\title{
The Role of Diagnostic Medical Imaging Techniques in the Evaluation of Perianal Fistula: A Review
}

\author{
Adityan R, BSc ${ }^{1}$ and Jerald Paul Immanue $2^{*}$ \\ ${ }^{1}$ Radiology and Imaging Technology, Sri Balaji Vidyapeeth University, India \\ ${ }^{2}$ Lab Specialist RMI Department, Institute of Applied Technology, UAE
}

*Corresponding author: Jerald Paul Immanuel, Lab Specialist RMI Department, Institute of Applied Technology, UAE, Tel: 050-488-2073

\begin{abstract}
The fistula in ano or perianal fistula is one of the common fistulae noted in routine surgical and clinical practice. It has the tendency to reoccur even after surgery, which is due to missed pathological tract or tissue during surgical exploration. An adequate pre-surgical mapping is a necessity for giving an excellent surgical outcome and choosing an appropriate surgical technique for treating various fistulas. So, medical imaging modalities like X-ray fistulography, endoanal ultrasonography, computed tomography fistulography, and the magnetic resonance fistulography were used for assessing fistula. Among these, magnetic resonance fistulography is the commonly preferred diagnostic medical imaging technique for interpreting the fistula as it has higher resolution on soft tissues, non-usage of ionizing radiation, which reduced the threat of radiation-induced cancer, and non-invasive imaging technique, which reduces patient discomfort and complications. The perianal region fistula is mainly classified into two types namely, park's classification and St. James university hospital's classification. Hence in this literature, we have reviewed the importance of medical imaging modality in interpreting perianal fistula, especially magnetic resonance fistulography, as an accurate preoperative, post-operative, and follow-up medical imaging modality for the perianal fistula's imaging. The subtraction technique, dynamic contrast imaging, volume imaging, and percutaneous instillation of aqueous jelly into the perianal fistula tract are the latest advances in imaging perianal fistula.
\end{abstract}

\section{Keywords}

Magnetic resonance imaging, Perianal fistula, Aqueous jelly, MRI contrast media

\begin{abstract}
Abbreviations
MRI: Magnetic Resonance Imaging; EAUS: Endo Anal Ultrasonography; CT: Computed Tomography; EUA: Examination Under Anaesthesia; MSCT: Multi Slice Computed Tomography; T: Tesla; SNR: Signalto Noise Ratio; FOV: Field of View; NSF: Nephrogenic Systemic Fibrosis; STIR: Short Tau Inversion Recovery; DCEMR: Dynamic Contrast Enhanced Magnetic Resonance Imaging; DWI: Diffusion Weighted Imaging, 3D: Three Dimension; MPR: Multi Planar Reconstruction; MIP: Maximum Intensity Projection
\end{abstract}

\section{Introduction}

The perianal region fistula is a pathological tract extending between the perianal skin and anal canal, commonly encountered in routine surgical practice [1]. Generally, a fistulous tract is a tract connecting one or two surrounding anatomical structures to the external skin surface, and it is said to be a sinus fistula tract when it connects only one hollow organ or skin [2]. The perianal fistula occurs either as tuberculosis, or one of the components of the inflammatory bowel diseases, such as Crohn's disease, localized infections of the anal crypts and glands, crypto glandular disease, trauma, and infection which are more complicated for treatment in $10 \%$ of the cases $[1,3]$. Among the general population, Crohn's disease occurs in 3 to 7 cases in 100,000 peoples [4]. Approximately $40 \%$ of the patients diagnosed with Crohn's disease will develop a perianal fistula, and $36 \%$ of patients having Crohn's disease present with a perianal fistula as their initial complaint

Citation: Adityan R, Immanuel JP (2021) The Role of Diagnostic Medical Imaging Techniques in the Evaluation of Perianal Fistula: A Review. Int J Radiol Imaging Technol 7:084. doi.org/10.23937/25723235.1510084

Accepted: December 13, 2021; Published: December 15, 2021

Copyright: (C) 2021 Adityan R, et al. This is an open-access article distributed under the terms of the Creative Commons Attribution License, which permits unrestricted use, distribution, and reproduction in any medium, provided the original author and source are credited 
in which $75 \%$ of the cases were complex [5]. The fistula in ano occurs approximately in 10 of 100,000 persons, with male predominance [6]. The male-to-female ratio of perianal fistula occurrence is 2:1 with a common symptom of discharge from the fistulous tract and pain due to the inflammatory tissues in $65 \%$ of the cases [7]. The reason for male predominance may be due to the higher abundance of anal glands [2]. The perianal fistulous tracts are more complex in $5-15 \%$ of cases with the presence of secondary tracts and extensions like an ischiorectal abscess, horseshoe fistula, and supralevator abscess outside the anal sphincter complex [8]. The perianal fistula's characteristics include the external and internal openings, primary tract, lateral ramifications from the main tract, and other diseases complicating the fistula [9].

Surgical intervention is the commonly preferred treatment for fistula in ano. But during surgery, improper excision lead to recurrences and over-excision may cause anal sphincter incontinence [1]. Up to $25 \%$ of fistulas reoccur, especially if secondary fistulous extension from the primary tract is present as it may be missed during surgery [10]. In a hospital in the United Kingdom, there were 12,000 patients affected by perianal fistula between the years 2008 to 2009 and 10,000 patients were treated surgically. Also, there were incident reports from the United States of America with a perianal fistula incidence rate of $0.18 \%-0.69 \%$ [11]. An excellent surgical treatment is associated with the perianal fistula's accurate preoperative evaluation because of its higher tendency to reoccur $[3,12,13]$. Society of American colon and rectal surgeons has formed a task force for the classification of perianal fistula as simple and complex in which complex type is a high risk factor of developing incontinence after surgery [9]. The pre-operative investigation of the pathological extension with medical imaging methods paved the way to the surgical elimination of all pathological tissues, thereby decreasing the recurrence rate [3]. Pre-operative imaging is mandatory for patients with a high probability rate of developing complex fistula or recurrent perianal fistula [5].

The imaging method is used to outline all the minute and hidden tracts along with its extension and relation to the anal sphincter complex [2]. The diagnostic evaluation of perianal fistula is challenging even for specialists in colorectal surgery and also for radiologists [4]. The initial role of radiologists is to be accurate and detail in making their final reports in estimating the perianal fistulous tract, as diagnostic information will be vital in making decisions about choosing whether medication or surgical intervention [13]. So, it is fundamental to see the perianal fistula's course along with its relevant findings before performing the surgical intervention [3]. Magnetic Resonance Imaging (MRI) is predominantly preferred for diagnosing the anal region fistula, anovaginal, and rectovaginal fistula's anatomy
[3]. MRI is preferred over endo anal ultrasonography (EAUS) and computed tomography (CT) due to its higher contrast resolution [14]. The pre-operative MRI of the anal fistula was mostly preferred since Lunniss, et al. reported that it accurately classified the fistulas in 14 (88\%) of 16 patients [15].

The MRI was highly accurate in classification and identification of perianal abscesses and horseshoe fistulous tracts, which guides during the surgical treatment by providing a pre-surgical map of the disease's extent that allows the physician to choose the appropriate surgical intervention [16]. The recommendations from the American Association of Gastroenterology states that for diagnosing the simple and complex types of perianal fistula, physical examination with rectosigmoid endoscopy is sufficient but in case of pain or planning for surgery as the initial treatment, examination under anesthesia (EUA) or MRI fistulography are preferred. Also, the recommendations acknowledge that performing EUA alone is not $100 \%$ accurate [17]. Hence, MRI is a considerable imaging modality for effective evaluation of fistula and its extension to peripheral areas [18].

\section{Grading of Perianal Fistula}

There are two types of perianal fistula classification [7]. They are:

\section{Park's classification}

St. James university hospital's classification

\section{Park's classification}

The Parks type of classification for perianal region fistula was formed by Parks, et al. from the results of 400 perianal fistula cases treated in St. Mark's hospital, London. There were four major types based on its extent and anal sphincter relationship, which is mainly developed for surgical usage $[7,13,19]$. The park's classification of the perianal fistula is given in Table 1.

\section{St. James University hospital's classification}

The radiologists formed the St. James university hospital's classification for being accurate and descriptive in their final reports to help surgeons in their treatment [7]. Morris, et al. reported that St James's University Hospital classification's grade 1 and grade 2 fistula types have satisfactory outcomes whereas grade 3 to grade 5 have unsatisfactory results as it needs a

Table 1: Park's type of perianal fistula classification.

\begin{tabular}{|l|l|}
\hline \multicolumn{2}{|l|}{ Park's Classification [9] } \\
\hline Type & Name \\
\hline I & Intersphincteric Fistula \\
\hline II & Transphincteric Fistula \\
\hline III & Suprasphincteric Fistula \\
\hline IV & Extrasphincteric Fistula \\
\hline
\end{tabular}


Table 2: St. James university hospital's classification of perianal fistula.

\begin{tabular}{|l|l|}
\hline \multicolumn{2}{|c|}{ St. James University Hospital's Classification [7] } \\
\hline Grade & Name \\
\hline I & Simple Linear Intersphincteric Fistula \\
\hline II & $\begin{array}{l}\text { Intersphincteric Fistula with an abscess or } \\
\text { secondary tract }\end{array}$ \\
\hline III & Transphincteric Fistula \\
\hline IV & $\begin{array}{l}\text { Transphincteric Fistula with an abscess or } \\
\text { secondary tract }\end{array}$ \\
\hline V & Supralevator and Translevator disease \\
\hline
\end{tabular}

surgical intervention [20]. The St. James university hospital's classification is given in Table 2.

\section{Medical Imaging Techniques}

The diagnostic medical imaging modalities used for preoperative mapping of the perianal fistula are [11],

$>$ Conventional fistulography

> Computed Tomography

$>$ Endo anal ultrasonography

> Magnetic Resonance Imaging

The conventional fistulography has sensitivity, and specificity ranges from $16 \%-87 \%$ [11]. It is assumed that CT fails to detect fistulous tracts along with its secondary extensions [11]. The EAUS and 3\% hydrogen peroxide instillation are accurate and preferable in perianal fistula evaluation [12]. The conventional fistulography, CT, and EAUS are inferior to MRI imaging [16]. Hence MRI replaces the examination under anesthesia (EUA) and is considered as the "gold standard" for preoperative perianal fistula evaluation [2].

\section{Conventional fistulography}

The conventional fistulography was the imaging technique used for perianal region fistula evaluation with radio-opaque contrast media instillation. This procedure is done by catheterizing the fistula's external opening with a cannula and injecting water-soluble contrast media into it, and obtaining a radiograph [18]. But the technique is accurate only in $16 \%$ of patients [8] because of the drawbacks listed below:

$>$ The difficulty in interpreting the secondary tract extension is due to the lack in filling of contrast media $[7,18]$.

$>$ The inability to visualizing the levator muscle to estimate the supralevator or infralevator extension [18] and the perianal fistula's relationship with anal sphincters [7].

\section{Computed tomography}

$\mathrm{CT}$ is performed both with post-instillation of rectal and intravenous contrast media instillation into the fistulous tract for perianal region fistula evaluation
[7]. Ergen, et al. has shown the rectal contrast media's effectiveness following the act of forced defecation to visualize the openings of the anovaginal fistula or rectovaginal fistula interior of the rectum [3]. CT usually fails to visualize inflammatory disease and also perianal abscess as it lacks soft-tissue resolution $[7,8]$ and also fails in correct perianal fistula's classification as it fails to show the tract near the sphincter ani externus muscle and levator muscle, which results from the similar attenuation values [21,22]. On MRI and EUS, the abscess that contains air may be difficult to interpret from bowel air, but CT is free from this limitation [14].

The Multislice computed tomography (MSCT) enables the acquisition of thin slices with excellent temporal resolution [14]. There are concerns raised regarding the $\mathrm{CT}$ usage as it may steer the chance of developing radiation-induced malignancies; however the advancements in CT's software and hardware configurations enable lesser radiation dose scans without compromising the image quality. The $\mathrm{CT}$, as well as MRI, have different advantages in perianal fistula evaluation, and CT will be the initial choice for non-complicated cases because of its inexpensiveness compared to MRI and wide range of availability. In some studies $\mathrm{CT}$ also provides comparable diagnostic information with MRI for the planning of surgery [14]. In obscured or negative $\mathrm{CT}$ findings, an MRI is necessary for further evaluation [22]. Hence adequate knowledge in choosing the appropriate modality for a specific pathology is necessary.

\section{Endo anal ultrasonography}

EAUS was the first medical imaging technique used for interpreting the anal sphincter complex's anatomy [3], rectal wall, and fistula with its relation to the sphincter complex $[7,8]$. The high-frequency transducers were used to accurately identify of intersphincteric fistula and the internal opening of the fistula [11]. The digital rectal examination and MRI is compared with EAUS in terms of correct perianal fistula classification, which resulted in $61 \%$ accuracy for a digital examination of rectum, $81 \%$ accuracy for EAUS, and $91 \%$ accuracy for MRI [7]. Also, for assessing the fistula's internal opening, EAUS shows $91 \%$ accuracy, and MRI shows $97 \%$ accuracy [7]. Nevertheless, EAUS has a major drawback of limited field of view (FOV), which disables its use in evaluating the superficial fistula, suprasphincteric fistula with secondary branches, extra sphincteric fistula, and fistula extending to some distance far away from the EAUS probe favoring the use of MRI in such cases [7,11]. Even though MRI is higher in every aspects, EAUS is preferred in most cases, especially in claustrophobia patients $[2,7,11]$.

\section{Magnetic resonance imaging}

MRI has beenappliedforvariousclinicalinvestigations, whereas its use on the evaluation of perianal fistulas 
was first reported in the 1990s with a higher rate of specificity and sensitivity [11]. The diagnostic accuracy reports of MRI in detecting perianal fistula were from 1992, and the fistula classification was from 1994 [8]. Surgical intervention is considered the preferable technique or reference standard for evaluating and treating fistula but, MRI is highly accurate than surgery for diagnostic evaluation for perianal fistula and Crohn's disease [1]. For selecting appropriate surgical technique and monitoring the fistula's extent, MRI is the choice as it clearly shows the pathological extension and also helps in prognosis with higher accuracy [2]. The MRI's advantage is its better visualization of anal sphincter complex and fistula relationship with multiplanar imaging technique and higher soft-tissue resolution [22].

The radiologist's role while interpreting the perianal fistula is to give detailed reports of the anal sphincter and fistula's relationship, enabling the surgeons to perform the appropriate surgical treatment that significantly reduces the recurrence and possible surgical complications like fecal incontinence [7]. $75 \%$ of the surgery with MRI as the pre-operative investigation reduces the recurrence of perianal fistula. So, patients with complaints of recurrent fistula should undergo MRI [10]. MRI has the ability in demonstrating sepsis (i.e., pus) in intersphincteric space, which is not found in probing that may cause recurrence $[6,7]$. The diagnostic information from MRI is considered a powerful predictor than surgery and post-operative outcome [7]. Villa C, et al. demonstrated the MRI's accuracy in ruling out the main fistulous tract with $100 \%$ sensitivity and $86 \%$ specificity, whereas for abscesses visualization, it gives $96 \%$ sensitivity and $97 \%$ specificity [19]. The MRI fistulography has a $95-97 \%$ sensitivity and specificity of $80-100 \%$ [7,22]. The instillation of MRI contrast media or sterile water improves the fistulous tract's delineation, which is impossible in patients with no external opening [23]. West, et al. investigated the comparison of endo anal MRI, surgery, and hydrogen peroxide enhanced EAUS. EAUS had a concordance rate of $81 \%$ with surgical findings, whereas MRI and surgery have $90 \%$ concordance [5]. Most of the perianal fistula cases were presented in the lower pelvic region, and in the case of supralevator fistula extension, bowel peristalsis may cause artifacts which can be overcome by anti-peristaltic agents [2].

$\mathrm{MRI}$ is the accurate pre and post-operative imaging technique in evaluating the fistulous tract with secondary extensions, but it has drawbacks like higher time consumption, higher expense, and claustrophobia in some patients $[14,15]$. This can be overcome by the use of a high field superconducting magnet (i.e. 3 tesla (T) MRI scanner), which gives better signal-to-noise ratio (SNR), increased spatial resolution that improves the lesion's visibility, increased temporal resolution, and reduced scan time [7]. Another crucial drawback of
$\mathrm{MRI}$ is that nerve and vasculatures can be mistaken as a fistulous tract [24]. MRI with high-resolution subtraction technique is an emerging trend for detecting perianal fistula, which is essential for surgery and has a higher preference from the gastroenterologists and surgeons with lesser patient discomfort [4].

\section{Coil selection}

The radiofrequency coils utilized for fistula in ano imaging are body coil; in some studies, spine coil, and endoanal coil [8]. Researchers have performed a comparative evaluation of body coil and endo anal coil MRI imaging, in which body coil MRI shows a surgical concordance rate of $96 \%$ and endo anal MRI shows $68 \%$ [8]. The radiofrequency body coil isplaced around the pelvic region for imaging. An MRI endo anal receiver coil with diameter a of $12 \mathrm{~mm}$ surrounded by rubber sheath is adequate for demonstrating inflammatory diseases with the FOV and is positioned in the patient's anal canal in lateral decubitus position. Later the patient's position is altered in supine, and an external clamp is used in immobilizing the coil for the entire scan period [25].

De Souza, et al. has shown the usage of an endoanal MRI coil to obtain high-resolution anal musculature and perianal fat images with $100 \%$ accuracy in detecting the fistulous tracts and abscess [24]. Stoker, et al. have demonstrated that the usage of endoanal MRI coil is superior in detecting cryptoglandular fistulas compared to body coil [24]. Simultaneously, Halligan and Bartram have shown the superior surgical concordance of body coil MRI with 17-18 tracts compared to endo anal MRI with 15-18 tracts [24]. MRI with endo anal coil was hoped as an advanced MRI imaging method as it gives high-resolution anatomical details of the sphincters, but it is not suitable for all the patients due to poor tolerance [6]. However, endo anal MRI provides good visualization of the internal opening, the limited FOV, and higher cost are a disadvantage of it $[8,12]$. Both coils can also be simultaneously used to get better diagnostic accuracy, but it is not easy to employ [2].

\section{Planning [7]}

In order to attain an exemplary sequence of planning, a T2-weighted sagittal plane image is acquired first, which shows the entire pelvic overview, anal canal's extent, and axis. The axial and coronal plane images were planned on the long axis of the anal canal in the sagittal plane to acquire true axial and coronal images, which is crucial for the perianal fistula's accurate evaluation. To avoid recurrences by the missed pathology on imaging technique, it is optimal to add the perineum and levator plate to rule out infected tracts and sepsis areas during planning.

\section{Pulse sequences}

MRI has come out as the commonly preferred 
imaging technique for ano vaginal fistula, rectovaginal fistula, and perianal fistula evaluation [26]. The tracts with enhancement in their walls are considered active tract, and non-enhancement in their walls were chronic tract [22]. Sometimes the active type of fistulous tract is surrounded by a chronic fibrous wall especially in repeated surgeryhistory [14]. The primary imaging sequence is the T2 weighted pulse sequence, which shows necessary diagnostic information on the fistula's location, especially in the axial plane [19] with the pelvic floor and anal sphincter complexes [2]. The tract in functional condition and abscess with pus filling shows hyper enhancement in T2 weighted pulse sequence along with the fluid and active inflammation differentiation $[5,7]$. Mostly the researchers prefer the T2 weighted turbo spin echo imaging method for appreciable differentiation between a hypo intense fibrous wall and hyper-intense fluid of the fistula, which collaterally enables nice visualization in the anal sphincter's layers [5].

The T1 weighted pulse sequence in fistulography is used for an anatomical overview of the pelvis, especially the ischiorectal fossae, levator ani plate, and sphincters. The anatomical structures are hard to interpret between the fistula tract, abscess, and inflammation as it has a lesser signal intensity, which is well demonstrated in the T2 weighted pulse sequence [6]. On T1 weighted imaging, the hemorrhage caused by postoperative intervention, and placement of fat-containing grafts after restorative surgery shows hyper enhancement which is distinguished from the residual fistulous tract $[2,6,7]$.

MRI uses gadolinium-based contrast media to give hyper enhancement of the active fistulous tract and abscess, which can be well differentiated from the old tracts showing hypo intense signal [27]. The use of contrast media increases the cost of the entire examination and is contraindicated for patients with higher renal function test values taking into the consideration of developing nephrogenic systemic fibrosis (NSF) [28]. In some hospitals, contrast-enhanced imaging was added in the regular MRI fistulography protocol for better visualization of the perianal fistula's anatomy [28]. The post-contrast instilled images were acquired in the $\mathrm{T} 1$ weighted fat suppressed sequence. The contrast-enhanced T1 weighted imaging shows a hypointense signal from fluid or active inflammation and shows a hyperintense signal from granulation tissue $[5,7]$. The hyper-enhancement after contrast media injection is because of the higher perfusion in tissues and vascular permeability [26]. The post-contrastacquired images were used to evaluate the degree of inflammation and differentiate the scar tissue from granulation tissues, increasing the diagnostic confidence prior to surgery [20].

The T1 weighted spoiled gradient contrast-enhanced pulse sequence provides higher resolution images with fair visibility of lateral ramifications and fistula extensions [21]. To rule out the position of the set on after surgery, T1 weighted gradient echo imaging sequence was used [20]. The chronic fistulous tracts show low enhancement in T1, T2 weighted imaging, and no enhancement in post-contrast $\mathrm{T} 1$ weighted images [7].

The fat suppression pulse sequence is not only used in T1 weighted imaging but also used in T2 weighted imaging or short tau inversion recovery (STIR) for distinguishing fluid and inflammatory changes [18]. The T2 weighted fat-suppressed image shows hyper enhancement in fluid, pus collection, and granulation tissue with hypo-intense fat background [13]. The STIR pulse sequence is a combination of both structural delineation and fat suppression, which influences mostly all the researchers to prefer it $[2,25]$. The sequence can also be performed in any one plane to effectively visualize the fluid channels clearly as it enhances longer T1 elements and T2 elements in a lesion $[2,25]$. STIR sequence has a disadvantage of decreased spatial resolution compared to frequency selective fatsaturated T2 weighted image, and in some of the cases, pseudo enhancement of inactive and fibrosed tracts leading to misinterpretation $[6,7,27]$. Nevertheless, for homogenous fat suppression, STIR is highly preferable to frequency selective fat-saturated $\mathrm{T} 2$ weighted image. In a study of 42 patients, the secondary tracts failed in showing the minute perianal abscess in it by using STIR sequence, which makes it less preferable than T1 weighted post-contrast imaging [7]. Frequency selective spectral pre-saturation and STIR sequence have longer scan time compared to traditional spin echo sequence. Hence, dynamic contrast-enhanced MRI imaging (DCEMR) is preferred over STIR in evaluating active sepsis with lesser scan time [8]. For the effective fistulous tract evaluation in abscess and sepsis, a combination of T2, STIR, and post-contrast T1 weighted imaging in axial as well as coronal planes were preferred [19].

The diffusion-weighted imaging (DWI) increases the confidence level of radiologists in reporting [22]. The mechanism of DWI is to image the water mobility changes because of the interaction with macromolecules, cell membrane, and environmental tissue alteration. So, inflammatory tissues tend to have hyper-enhancement in DWI. The DWI is combined with T2 weighted imaging in improving the diagnostic efficiency of patients with contraindication of contrast media imaging, but it needs an extra 2-4 minutes of scan time [7,28]. The DWI sequences were added to the routine MRI fistulography protocol in many institutions, which is not tricky for implementation [28]. DWI's greatest disadvantage is poor spatial resolution compared to spin echo and gradient echo pulse sequences [28].

The Three dimensional (3D) imaging had paved the 
way for multiplanar reconstruction (MPR) into any plane, eventually decreasing the routine number of pulse sequences performed [7]. The gradient echo 3D imaging enabled post-processing techniques like MPR, maximum intensity projection (MIP), and T2 weighted 3D turbo spin echo imaging provides raw data for any desired plane reconstruction $[4,14]$. The advantage of using a 3D pulse sequence was [7]:

$>$ No operator dependence as any desired plane can be reconstructed.

$>$ Higher signal to noise ratio.

Thinner slice acquisition in a zero-gap.

However, the T2 weighted 3D imaging is not preferred in some of the cases as it takes a longer time for a single sequence and more sensitive to patient motion [14].

\section{Current trends}

There are numerous perianal fistula imaging trends like additional pulse sequences, advanced postprocessing techniques, alternative instillation media, etc. The advanced acquisition technique is volume imaging with isotropic resolution, a simple innovation technique that enables $3 \mathrm{D}$ reconstruction in a lesser scan time as only one image plane is acquired [3]. The latest diagnostic technique for a perianal fistula is digital subtraction MRI done by acquiring T1 fat-saturated pre and post-contrast sequences. The acquired images were subtracted, which gives dark fat background and hyper enhanced fistulous tract with different degrees of low signal intensity of fluids, which enables reasonable interpretation of minute fistulous tracts $[4,7]$.

The DCEMR is used to evaluate Crohn's disease's activity rate by acquiring time signal intensity curves from a T1 weighted image for active tract estimation by measuring the volume of enhancing pixels [7]. This is used to select a subpopulation of Crohn's disease patients who needs close monitoring as the disease may be extensive in the future $[7,26]$. Hence DCEMR is considered a promising technique in predicting the post-operative outcome [29].

Another technique for diagnosing perianal fistula is using aqueous jelly instead of gadolinium-based contrast media and saline. Plain MRI cannot delineate the walls of the fistulous tract, particularly the smaller tract. So, there is a need for instillation media for tract delineation. Initially, gadolinium contrast media and saline were used by Wanicczek, et al. for better visibility of the pathological tract. The contrast media instillation via rectum is also preferred for imaging [18]. Nevertheless, gadolinium contrast media and saline instilled directly into the tract gives temporary distension as it has watery consistency, disabling the minute tract's delineation, identifying the lateral ramification and internal opening's position. The gadolinium contrast media also have a disadvantage of giving false positive and false negative results and increasing the total cost of the examination. Hence a modified innovative technique is the percutaneous instillation of the aqueous jelly into the perianal fistulous tract to diagnose perianal fistula. This technique elevates the diagnostic evaluation by distending the fistulous tract and inherent contrast due to longer $\mathrm{T} 2$ relaxation time, which collaterally eliminates the need of MRI contrast media. The aqueous jelly is a cheap, viscous, inert, and readily available, that can be routinely used to give sustained and adequate delineation of the tracts [1].

\section{Treatment and Management}

\section{Surgical intervention}

The estimation of a successful surgical intervention is the correct balance of disease eradication and maintaining the normal function, which is achieved by detailed knowledge about the fistula's relation with the anal sphincter and secondary extensions from it to avoid recurrences and incontinence $[3,18,30]$. After surgical intervention, clinical examination is difficult as fibrosis takes place; in such cases, MRI is referred instead of endoanal ultrasonography as it also distinguishes active sepsis from fibrosis [17]. The types of surgical intervention are $[12,20]$ :

\section{Fistulotomy}

\section{Fistulectomy}

Seton placement, etc.

\section{Fistulotomy [20]}

Fistulotomy is done in treating low transphincteric and intersphincteric fistula as it maintains fecal continence [5]. The surgery is performed by an initial incision in the fistula's opening and blending it with the anal canal for healing process. But performing this type of surgery for treating complex fistula leads to incontinence in approximately $50 \%$ of the cases, which is overcome by marsupialisation of fistulotomy wounds to accelerate the healing process.

\section{Fistulectomy [20]}

Fistulectomy is a procedure for treating low transphincteric and intersphincteric fistula. The surgery is performed by completely removing the pathological tract causing larger wound compared to fistulotomy that is overcome by marsupialisation of the wounds to accelerate the process of healing.

\section{Noncutting seton [20]}

It is an alternative technique for fistulotomy and fistulectomy with non-healing wounds. Setons are threads made up of nylon, silk, rubber, polyester, plastic, silicone, self-locking cable ties, and wire. It prevents the closing of the fistula's external opening site, thereby allowing the drainage of fluids to prevent 
abscess formation, which is the primary or temporary treatment for reducing the fistula's severity [7].

\section{Advances in surgical intervention [20]}

Biomaterials, which are a biological glue with collagen materials, are used recently as an alternative treatment method for perianal fistula with an initial success rate in $60-85 \%$ of cases. The biological glue used is a fibrin glue that has both thrombin and fibrinogen components to make it a tissue adhesive. The procedure starts the instillation of both components into the tract to seal it by forming a fibrin clot, which has a higher healing rate with delayed recurrences.

\section{Medication}

Crohn's disease has higher healthcare and hospitalization costs [23], which are treated initially by antibiotics like ciprofloxacin and metronidazole [5]. The antibodies with anti-tumor necrosis factor like infliximab are used for good results [7]. The infliximab treatment for every eight weeks reduces $70 \%$ of surgeries and hospitalization for Crohn's disease patients and 50\% reduction in surgical procedures compared to placebo maintenance treatment [23].

\section{Conclusion}

The perianal fistula imaging is most challenging as any missed diagnostic information may lead to recurrences and repeated surgical intervention. The most commonly preferred investigation for perianal region fistula evaluation is MRI fistulography, which is considered as the accurate pre-operative, postoperative, and follow-up imaging technique. However, EAUS is also preferred next to MRI as it demonstrates the pathological tract with appreciable accuracy. The most significant advantage of MRI is increased soft tissue anatomy, which is very important in evaluating fistulous tract extension and non-usage of ionizing radiation that reduces the chance of radiation-induced cancer occurrence. The aqueous jelly administration, instead of MRI contrast media into the perianal fistulous tract provides appreciable delineation of the fistulous tract and detection of the primary fistulous tract with an internal opening.

\section{Acknowledgement}

Nil.

\section{Sources of Support}

Nil.

\section{Statement of Equal Authors' Contribution}

We accept the submission of this article and are responsible for its content.

\section{References}

1. Aggarwal R, Soni BK, Kumar JU, George RA, Sivasankar $R$ (2017) MR fistulography with percutaneous instillation of aqueous jelly: A cost effective technique innovation. Indian J Radiol Imaging 27: 161-166.

2. Torkzad MR, Karlbom U (2010) MRI for assessment of anal fistula. Insights Imaging 1: 62-71.

3. Yildirim N, Gökalp G, Öztürk E, Zorluoğlu A, Yilmazlar T, et al. (2012) Ideal combination of MRI sequences for perianal fistula classification and the evaluation of additional findings for readers with varying levels of experience. Diagnostic Interv Radiol 18: 11-19.

4. Schaefer O, Lohrmann C, Langer M (2004) Assessment of Anal fistulas with high-resolution subtraction MRfistulography: Comparison with surgical findings. J Magn Reson Imaging 19: 91-98.

5. Ziech M, Felt-Bersma R, Stoker J (2009) Imaging of perianal fistulas. Clin Gastroenterol Hepatol 7: 1037-1045.

6. Morris J, Spencer JA, Ambrose NS (2000) MR imaging classification of perianal fistulas and its implications for patient management. Radiographics 20: 623-635.

7. Criado $\mathrm{J}$ de $\mathrm{M}$, del Salto LG, Rivas PF, del Hoyo LFA, Velasco LG, et al. (2012) MR imaging evaluation of perianal fistulas: Spectrum of imaging features. Radiographics 32: 175-194.

8. Beets-Tan RGH, Beets GL, Van der Hoop AG, Kessels AGH, Vliegen RFA, et al. (2001) Preoperative MR imaging of anal fistulas: Does it really help the surgeon? Radiology 218: $75-84$

9. Konan A, Onur MR, Özmen MN (2018) The contribution of preoperative MRI to the surgical management of anal fistulas. Diagnostic Interv Radiol 24: 321-327.

10. Buchanan G, Halligan S, Williams A, Cohen CRG, Tarroni $D$, et al. (2002) Effect of MRI on clinical outcome of recurrent fistula-in-ano. Lancet 360: 1661-1662.

11. Siddiqui MRS, Ashrafian $H$, Tozer $P$, Daulatzai N, Burling D, et al. (2012) A diagnostic accuracy meta-analysis of endoanal ultrasound and MRI for perianal fistula assessment. Dis Colon Rectum 55: 576-585.

12. Waniczek D, Adamczyk T, Arendt J, Kluczewska E, Kozińska-Marek E (2011) Usefulness assessment of preoperative MRI fistulography in patients with perianal fistulas. Polish J Radiol 76: 40-44.

13. Mohey N, Hassan TA (2017) Effectiveness of magnetic resonance imaging in grading of primary perianal fistula and its associated findings in correlation with surgical outcome. Egypt J Radiol Nucl Med 48: 1-6.

14. Liang C, Jiang W, Zhao B, Zhang Y, Du Y, et al. (2013) CT imaging with fistulography for perianal fistula: Does it really help the surgeon? Clin Imaging 37: 1069-1076.

15. Buchanan GN, Halligan S, Bartram Cl, Williams AB, Tarroni D, et al. (2004) Clinical examination, endosonography, and MR imaging in preoperative assessment of fistula in ano: Comparison with outcome-based reference standard. Radiology 233: 674-681.

16. Mullen R, Deveraj S, Suttie SA, Matthews AG, Yalamarthi $S$ (2011) MR Imaging of fistula in ano: Indications and contribution to surgical assessment. Acta Chir Belg 111: 393-397.

17. Sahni VA, Ahmad R, Burling D (2008) Which method is best for imaging of perianal fistula? Abdom Imaging 33: 26-30.

18. Halligan S, Stoker J (2006) Imaging of fistula in ano. Radiology 239: 18-33.

19. Daabis N, El Shafey R, Zakaria Y, Elkhadrawy O (2013) 
Magnetic resonance imaging evaluation of perianal fistula. Egypt J Radiol Nucl Med 44: 705-711.

20. Gage KL, Deshmukh S, Macura KJ, Kamel IR, Zaheer A (2013) MRI of perianal fistulas: Bridging the radiologicalsurgical divide. Abdom Imaging 38: 1033-1042.

21. Mazroa JA, Elmogy SA, Elgendy MM (2012) Value of contrast enhanced spoiled gradient (SPGR) MR and MIP MR imaging in diagnosis of peri-anal fistula. Egypt $J$ Radiol Nucl Med 43: 119-128.

22. Soker G, Gulek B, Yilmaz C, Kaya O, Arslan M, et al. (2016) The comparison of CT fistulography and MR imaging of perianal fistulae with surgical findings: A case-control study. Abdom Radiol 41: 1474-1483.

23. Lichtenstein GR, Yan S, Bala M, Blank M, Sands BE (2005) Infliximab maintenance treatment reduces hospitalizations, surgeries, and procedures in fistulizing Crohn's disease. Gastroenterology 128: 862-869.

24. Mahjoubi B, Haizadch Kharazi H, Mirzaei R, Moghimi A, Changizi A (2006) Diagnostic accuracy of body coil MRI in describing the characteristics of perianal fistulas. Color Dis 8: 202-207.
25. Szyszko TA, Bush J, Gishen P, Sellu D, Desouza NM (2005) Endoanal magnetic resonance imaging of fistulain-ano: A comparison of STIR with gadolinium-enhanced techniques. Acta Radiol 46: 3-8.

26. Horsthuis K, Lavini C, Bipat S, Stokkers PCF, Stoker J (2009) Perianal Crohn disease: Evaluation of dynamic contrast-enhanced MR imaging as an indicator of disease activity. Radiology 251: 380-387.

27. Beckingham IJ, Spencer JA, Ward J, Dyke GW, Adams C (1996) Prospective evaluation of dynamic contrast enhanced magnetic resonance imaging in the evaluation of fistulain ano. Br J Surg 83: 1396-1398.

28. Hori M, Oto A, Orrin S, Suzuki K, Baron RL (2009) Diffusionweighted MRI: A new tool for the diagnosis of fistula in ano. J Magn Reson Imaging 30: 1021-1026.

29. Chapple KS, Spencer JA, Windsor ACJ, Wilson D, Ward $\mathrm{J}$, et al. (200) Prognostic value of magnetic resonance imaging in the management of fistula-in-ano. Dis Colon Rectum 43: 511-516.

30. Sangwan YP, Rosen L, Riether RD, Stasik JJ, Sheets JA, et al. (1994) Is simple fistula-in-ano simple? Dis Colon Rectum 37: 885-889. 